\title{
Transcutaneous Vagus Nerve Stimulation in Private Healthcare Center: A Small-Scale Investigation Targeting Anxiety, Irritable Bowel Syndrome and Chronic Pain
}

\author{
Pascal J.D. Grolaux \\ Department of Osteopathy, Kinesis Health Associates, 2d floor, 155 Ochterloney Street, Dartmouth, B2Y1C9, Nova Scotia, Canada.
}

Article Info

\section{Article Notes}

Received: May 28, 2019

Accepted: October 17, 2019

\section{${ }^{*}$ Correspondence:}

Dr. Pascal J.D. Grolaux, Department of Osteopathy, Kinesis Health Associates, 2d floor, 155 Ochterloney Street, Dartmouth, B2Y1C9, Nova Scotia, Canada; Telephone No.: +1(902)464-2225; Fax No: +1(902) 464-2244; Email: pgrolaux@kinesishealth.ca.

(C) 2019 Grolaux PJD. This article is distributed under the terms of the Creative Commons Attribution 4.0 International License

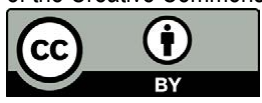

\section{Keywords:}

Transcutaneous Vagus Nerve Stimulation (Tvns)

Anxiety

Irritable Bowel Syndrome (IBS)

Chronic Pain

Private Healthcare Center

\section{Abstract}

Background: Generalized anxiety disorder (GAD), chronic pain (CP) and irritable bowel syndrome (IBS) are debilitating inflammatory disorders that are frequently seen in primary care facilities but not sufficiently addressed by classical medical treatments. Transcutaneous vagus nerve stimulation (tVNS) is a promising therapeutic tool for a wide range of conditions that showed already encouraging clinical results. However, the effects of tVNS, on these disorders, GAD, IBS and CP, were not yet explored in the context of an investigation conducted in a private healthcare center.

Objective: In an uncontrolled, open label, small investigation scale study, we investigated the feasibility, safety and the effects of tVNS for patients suffering from GAD, CP and IBS in a multidisciplinary healthcare center.

Methods: The effects of tVNS on GAD, CP and IBS in 10 participants (anxiety, 40\%; chronic pain, 30\%; IBS, 30\%) were investigated during a 4-week period and a 2-month follow-up. GAD, CP and IBS were assessed using the Generalized Anxiety Disorder GAD-7, the Brief Pain Inventory Short Form questionnaire and the Irritable Bowel Syndrome Severity Scoring System. Transcutaneous vagus nerve stimulation was performed using a transcutaneous electrical nerve stimulation device and ear clip electrodes plugged in the concha area of the ear. All participants, received a bi-weekly 30-minute stimulation for 4 weeks. The tVNS parameters, (GAD: $20 \mathrm{~Hz}-$ $80 \mu \mathrm{s}),(\mathrm{CP}: 5 \mathrm{~Hz}-200 \mu \mathrm{s}),(\mathrm{IBS}: 3 \mathrm{~Hz}-250 \mu \mathrm{s})$, were set for each group to target different physiological effects meditated by the vagus nerve.

Results: The anxiety and the IBS group showed a non-statistically significant improvement but an improved clinical status (mean score from "severe" to "moderate") both at the end of the stimulation period (4 weeks) and at 2-month follow-up. The CP group didn't show any significant clinical improvement (mean score from "moderate" to "moderate"). Furthermore, tVNS was demonstrated to be likely safe and was well tolerated.

Conclusions: Due to low sample size, this study failed to demonstrate significant clinical effects of tVNS on GAD, IBS and CP. However, trend analysis may carefully suggest tVNS to be a noteworthy clinical alternative to be used in private healthcare center in the treatment of chronic inflammation disorders like GAD and IBS. Acute tVNS was well-tolerated and is likely safe. Powerful, double-blind controlled studies are needed to support the use of tVNS for these disorders.

Clinical Trial Registration: http:/www.clinicaltrials.gov. Unique identifier: NCT03440255.

\section{Abbreviations}

$\alpha 7$ nAChR, alpha7 nicotinic acetylcholine receptor; ABVN, auricular branch of the vagus nerve; CAN, central autonomic network; CAP, cholinergic anti-inflammatory pathway; $\mathrm{CP}$, chronic pain; DMVN, dorsal motor nucleus of the vagus; GABA, $\gamma$-aminobutyric acid; GAD, general 
anxiety disorder; HR, heart rate; HPA, hypothalamic pituitary adrenal; HRV, heart rate variability; IBS, irritable bowel syndrome; IL, Interleukin; fMRI, functional magnetic resonance imagery; NF- $\kappa \mathrm{B}$, nuclear factor kappa $\mathrm{B}$; NTS, nucleus tractus solitarius; pNN50, percentage of successive R-R intervals that differ by more than $50 \mathrm{~ms}$ from the preceding one; PW, pulse width; RMSSD, root mean square of successive R-R interval differences; RR, respiratory rate; RSA, respiratory sinus arrhythmia; SD1, standard deviation of the distance of each point from the $y$ $=\mathrm{x}$ axis in a Poincaré plot; TENS, transcutaneous electrical nerve stimulation; TNF, tumor necrosis factor; VN, vagus nerve; nVNS, non-invasive vagus nerve stimulation; tVNS, transcutaneous vagus nerve stimulation.

\section{Introduction}

The vagus nerve (VN), the tenth cranial nerve, is the major component of the parasympathetic nervous system and plays a key role in the regulation of neuroendocrineimmune physiological and allostatic processes to maintain homeostasis through a complex integrated central and peripheral autonomic network, i.e., the activation of the hypothalamic pituitary adrenal (HPA) axis by its afferents (sensory), and the 'cholinergic anti-inflammatory pathway' (CAP) through its efferents (motor). This mixed nerve, described as the single most important nerve in the body, contains $80 \%$ afferent and $20 \%$ efferent fibers (80.000 to 100.000 fibers, non-myelinated and myelinated), although the right cervical portion of the $\mathrm{VN}$ is thicker than the left $^{1}$. The sensory vagal pathways terminating in the nucleus of the solitary tract convey visceral, somatic and taste sensations to the brain; including cardiorespiratory, gastrointestinal and immunological signals ${ }^{2,3}$. The motor efferent fibers, emerging from the nucleus ambiguus and the dorsal motor nucleus of the vagus (DMNV) in the medulla, project to several organs of the neck, the thorax and the abdomen including the organs of the reticuloendothelial system such as the liver and the spleen rich in macrophages activated by the inflammatory reflex ${ }^{4}$. Furthermore, the VN, through enteric neurons, he connects to the autonomic enteric nervous system, making the bidirectional gut-brain connection ${ }^{5}$.

Less than two decades ago, vagus nerve stimulation (VNS) had been approved in Canada, in the U.S. and in Europe for the treatment of multiple difficult-totreat conditions including drug- resistant epilepsy and depression $^{6-8}$. Implantable VNS device received CE Mark approval in 2001 for the treatment of mood disorders. Since the earliest report of VNS implantation in 1988, for drug-resistant epilepsy, tens of thousands of patients worldwide have received VNS therapy, and more than 100,000 patient-years of experience have been accrued 9 . There is new evidence of potential use of VNS as a therapeutic intervention for pathology such as obesity, pain management, cardiovascular diseases, diabetes, inflammatory bowel disease and other autoimmune diseases $^{10,11}$.

Transcutaneous vagus nerve stimulation (tVNS) is an emerging, promising alternative therapeutic tool that allows for the stimulation of the VN without the need of surgery. Transcutaneous auricular vagus nerve stimulation sends electrical impulses along the auricular branch of the $\mathrm{VN}(\mathrm{ABVN})$ that will trigger the same physiological effects as the invasive stimulation but without the side effects of surgery ${ }^{12-14}$. Indeed, in humans, high density of afferent fibers of the ABVN are found in the meatus acusticus externus cartilaginous and innervate $100 \%$ of the cymba conchae and $45 \%$ of the cavum of the outer ear ${ }^{15,16}$. The somatic sensory vagal afferents or Arnold's nerve represent a small fraction of the vagus, they transmit sensation from the concha to its termination center located in the nucleus tractus solitarius (NTS) ${ }^{2}$. The purpose of the tVNS to reach clinical efficacy is to selectively excite, by low-intensity current $(0,1$ to $1.8 \mathrm{~mA})$, the thick myelinated sensory $A \beta$-fiber in the $A B V N$, but not the $A \delta$ thin myelinated and C-fibers conveying pain ${ }^{17-19}$. Indeed, it was demonstrated that the vagal afferent C-fibers destructed by capsaicin do not alter the antiepileptic effect of $\mathrm{VNS}^{20}$. Safi et al. estimated that the ratio of A $\beta$-fiber in the ABVN compared to the cervical VN stimulated in invasive therapy was $\sim 1: 5$ and $\sim 1: 6$ on the left and right side, respectively. They concluded that Transcutaneous auricular vagus nerve stimulation might be a promising alternative to invasive VNS stimulation ${ }^{19}$. Several animals and functional magnetic resonance imagery (fMRI) human studies confirmed that the auricular fibers of the vagus nerveVN activated the NTS $^{13,18,21,22}$. These fibers projecting to the NTS will activate and modulate several simple and complex loops and circuitry (i.e., autonomic brainstem and forebrain loops), centrally in a bottom-up mode, and trough up-down reactions, the HPA axis and, in the periphery, immune tracts as the $\mathrm{CAP}^{23-25}$. Traditionally and otherwise indicated only the left branch of the ABVN is stimulated so that there is less influence on the heart rate, since the left VN innervates the atrioventricular node and the right VN innervates the sinoatrial node controlling the pace-making function of the heart ${ }^{26}$.

Recently, the booming of pre-clinical and clinical studies in bioelectronic medicine showed very encouraging results of tVNS in the field of psychiatry as well as in gastroenterology and in the management of chronic pain ${ }^{27-36}$.

\section{Anxiety}

In recent years high psychological distress disorders like anxiety and depression have increased dramatically, they are now one of the most common mental disorders with prevalence throughout the ages of 18 to 64 . In the 
United States only, 40 million people which represents more than $16 \%$ of the population are suffering from anxiety disorders ${ }^{37-39}$. General anxiety disorder (GAD) is characterized by both psychological and physical symptoms such as excessive anxiety, poor concentration, irritability, impaired social or occupational functioning, fatigue, muscle tension and poor sleep ${ }^{40}$.

Since Zabara, in 1985, demonstrated the antiseizure actions of vagal nerve stimulation in dogs, the effect of VNS in the treatment of epilepsy, depression and anxiety disorders gained in evidence ${ }^{41-44}$. Although the putative mechanisms behind the effects of VNS in anxiety are not clear, functional anatomy revealed that the vagus nerveVN, as a major component of the neuroendocrine-immune axis, leads to coordinate neural, behavioural, emotional, pain and endocrine responses that are critical for goaldirected behaviour and adaptability through central and peripheral neural reflex circuits ${ }^{23}$. For instance, short efferent vagovagal circuits will activate the cholinergic antiinflammatory pathway that will act on spleen macrophages to modulate innate autoimmune responses by reducing significant tumor necrosis factor (TNF) production and hence will trigger an effect on diseases with an inflammatory component ${ }^{45}$. Moreover, It was demonstrated that there is a significant link between stress disorders and people living with autoimmune conditions and neuroinflammation as a possible cause of anxiety disorders ${ }^{46}$. For instance, in a large adult cohort study, Vogelzangs studied the association between anxiety and inflammation and found that immune dysregulation was especially present in persons with a lateonset anxiety disorder. He showed evidence that elevated C-Reactive Protein level can be found among several common anxiety disorders ${ }^{47}$. On the other hand, direct or indirect longer efferent reflexes connected with the NTS will involve several mood-regulating limbic, subcortical and cortical brain regions as well as stimulation of the HPA axis that is the afferent arm of the anti-inflammatory reflex ${ }^{48,49}$. Imbalance of $\gamma$-aminobutyric acid (GABA) and glutamate signalling, i.e., low GABA and high glutamate, can contribute to excitotoxicity via inflammatory pathways. Indeed alterations in the kynurenine pathway from glial inflammation result in glutamate toxicity by blocking glutamate reuptake and injections of TNF- $\alpha$ and IL- 6 in the amygdala produce the same excitotoxicity ${ }^{50}$. Increased inflammatory cytokines that is classically associated with increased oxidative stress and sympathetic activity contributing to disrupt the synthesis of monoamines is commonly seen in patients with mood and anxiety-related disorders. Together with enhanced cytokine release, psychosocial stress frequently seen in anxiety disorders promote monocytic cell activation with increased expression of the transcription nuclear factor $\kappa \mathrm{B}(\mathrm{NF}-\kappa \mathrm{B})$.

Transcutaneous vagus nerve stimulation, as demonstrated by Zhao et al. in a rat model, decreased the serum levels of inflammatory cytokines such as TNF- $\alpha$, IL$1 \mathrm{~b}$, and IL-6, as well as the proinflammatory transcription factor such as NF-kB. These anti-inflammatory effects were not seen after vagotomy or administration of an $\alpha 7$ nicotinic receptor $(\alpha 7 \mathrm{nAChR})$ antagonist ${ }^{51}$. These actions of inflammatory cytokines ultimately are involved in alterations in the central autonomic network (CAN) including those related to basal ganglia, paraventricular and hypothalamic nuclei, prefrontal reward circuits, anxiety-related amygdala and insular circuitry ${ }^{52-54}$. Based on the central effects of VNS on brain areas involved in anxiety and on studies that reported its anxiolytic effects in patients treated for epilepsy and depression, non-invasive modulation of the $\mathrm{VN}$ could be a feasible alternative to classical pharmaceutical treatment in the management of anxiety disorders such as GAD.

\section{Irritable bowel syndrome}

Irritable bowel syndrome (IBS), which is a chronic functional gastrointestinal disorder that combines abdominal pain, frequent bloating, predominantly diarrhea or constipation, or both, is one of the most widely recognised functional bowel disorders and is mostly prevalent in women. More than $10 \%$ of the global adult population report symptoms compatible with the condition. In North America alone, it represents 30\% of reported consultation rate in primary care ${ }^{55-57}$. There is a growing awareness and evidence of a strong connection between the gut and the brain relaying functional alterations of the gastrointestinal tract to various central key structures in the brain. Anatomically,. mesenteric bundles of vagal afferent fibers innervates the intestines, distributing to all the layers of its wall ${ }^{58}$ and preganglionic neurons of vagal efferent originating in the DMNV are connected with postganglionic neurons of the enteric nervous system in the GI tract $^{59}$.

The vagus nerveVN is central in the bidirectional brain-gut-brain communication. Indeed, the activation of vagal afferents generates several coordinated responses (autonomic, endocrine, emotional and behavioral) via central pathways involving the dorsal vagal complex that turns back these responses into immune-endocrine reflexes, e.g., the HPA axis and CAP activation ${ }^{23,60}$. In irritable bowel syndrome (IBS) each level of the axis can be affected leading to gut dysfunction as well as the change in brain neuroplasticity involving the $\mathrm{CAN}^{61}$. Here again, imbalance of the ANS, high level of peripheral and central inflammation and oxidative stress play a major role in the physiopathology of this autoimmune disease ${ }^{62,63}$. The mechanism of the anti-inflammatory action of tVNS on the enteric system is independent of the spleen, therefore, the vagal anti-inflammatory input is direct to the gut $t^{5}$.Indeed, The et al. demonstrated that left cervical VN stimulation 
reduces small intestinal inflammation by CAP activation and prevents the development of postoperative ileus in mice ${ }^{64}$. They confirmed that the attenuation of macrophage activation following electrical vagal nerve stimulation was mediated by alpha7 nAChR- dependent STAT3 signaling in intestinal macrophages ${ }^{65}$.

Moreover, in a murine model of inflammatory bowel disease, Ghia et al. showed that the VN plays a counterinflammatory role in acute colitis via a macrophagedependent mechanism ${ }^{66}$. Recently, Bonaz et al. showed evidence of VNS as a safe and effective tool in the treatment of Crohn's disease ${ }^{67}$.

One possible mechanism of non-invasive VNS could start after activation of the NTS from the stimulation of the AVBN afferent fibers. In turn, the NTS would stimulate the dorsal motor nucleus of the vagus. The efferent vagus nerveVN stimulates cholinergic enteric neurons that are in close contact within the intestinal wall resident macrophages expressing $\alpha 7 \mathrm{nAChR}$. At this site, the activation of $\alpha 7 \mathrm{nAChR}$ will, in turn, result in the inhibition release of key proinflammatory cytokines. Therefore, the activation of myenteric cholinergic neurons via VNS may represent a non-pharmaceutical strategy to control immune-mediated intestinal inflammatory disorders ${ }^{31,68}$.

Based on the anti-inflammatory effects of the VNS (through the HPA axis and the CAP) and because the VN is a key element of the autonomic nervous system in the gut (microbiota)-brain relationship in inflammatory conditions of the gastrointestinal tract ${ }^{25,56,69}$, tVNS could be beneficial, as adjunctive therapy to conventional treatment, like probiotics, antibiotics and anti-depressant drugs ${ }^{70}$, and easily manageable in private healthcare facilities with rare serious side effects ${ }^{71}$.

\section{Chronic Pain}

There is no universally accepted definition for chronic pain, although it is often defined as pain that persists beyond the expected time of healing (usually 12 weeks) ${ }^{72}$. Indeed, when pain subsides for more than 3 months it becomes chronic and difficult to manage requiring complex pharmacotherapy and non-classical approaches ${ }^{73}$. This debilitating, difficult-to-treat condition, accounts for frequent physician visits and excessive medical evaluations, and is often associated with negative outcomes. Chronic pain is currently one of the most common and costly chronic health conditions in North America ${ }^{74,75}$.

Perception of pain is influenced by cognitive and emotional processes that constitute a complex emotional experience that varies significantly from one individual to the next ${ }^{76}$.

From animal experiments, VNS can also elicit both inhibition and facilitation of nociception, and in humans, treated by chronic VNS, recent physiologic researches demonstrated the influence of VNS on pain perception ${ }^{77}$. One of the mechanisms involved may be linked to the reduced production of inflammatory cytokines, like TNF- $\alpha$ by the spleen macrophages after $\mathrm{VNS}^{78}$, that are known to significantly induce C-fibers peripheral activation and central sensitization, e.g. hyperalgesia, allodynia, prolonged electrophysiological discharge, and an afterstimulus unpleasant quality of the pain ${ }^{79,80}$. For instance, injections of endotoxin administered directly into the brain produce changes in sensitivity to mechanical stimuli, and spinal intrathecal cytokines injection also leads to the development of allodynia and hyperalgesia ${ }^{81,82}$. Moreover, bilateral sub diaphragmatic vagotomy increases bradykinin-induced hyperalgesia and inflammation ${ }^{83}$. The brain is thus able to regulate sensitivity of nociceptors all over the body by a neuroendocrine mechanism and from the NTS, in a bottom-up neurosomes stimulation, to higher brain regions known to modulate the pain conveyed by, the rostral ventromedial medulla, the spinoreticulothalamic tract, gigantocellular reticular nucleus, periaqueductal grey matter, medial thalamus and anterior cingulate cortex ${ }^{23,84-86}$. From the stimulation of the AVBN, the alteration of vagal afferent activities in this supraspinal network of brain areas has been hypothesized to be the physiological substratum of VNS therapeutic effects ${ }^{13,87,88}$.

In addition to the anti-inflammatory properties of VNS, recent animal and human studies have shown analgesia and modulation of pain mediated by vagal afferents that inhibits central nociceptive reflexes and transmission through activation of key pain-network structure of the CAN like the NTS, locus coeruleus, periaqueductal grey matter, raphe magnus, thalamus and hypothalamus ${ }^{89,90}$. As noninvasive VNS showed evidence of significant encouraging results (safety, tolerability, efficacy) in human research for the treatment of fibromyalgia, chronic pain (CP), headaches and migraines and trigeminal allodynia ${ }^{91}$, we could possibly prescribe it as a complementary treatment to classical pharmacotherapy.

\section{Anxiety, IBS and CP}

Anxiety, IBS and CP are commonly seen in combination and related to each other, in functional disorders, as comorbidities, and they may be part of central sensitivity syndromes as well ${ }^{92-94}$. For instance, patients with higher self-rated pain might experience a greater degree of anxiety ${ }^{95}$. Moreover, headache, arthralgia, insomnia, and fatigue are extra-intestinal symptoms frequently reported by the patients in association with digestive disorders. Finally, chronic pain, like in fibromyalgia, is frequently associated with IBS $^{59}$. These conditions, and especially when linked to each other, are difficult to manage, and often are treated by a combination of pharmaceutical, psychological and non-conventional approaches ${ }^{96-98}$. 
A recent systematic review showed that non-invasive vagus nerve stimulation (nVNS) is very safe and extremely well tolerated in humans ${ }^{71}$. Therefore, given a better knowledge of the neuroanatomical and neuroendocrineimmunological effects of VNS its safety and therapeutic benefits in subjects suffering from mental health and inflammatory disorder, we aimed at evaluating the safety, tolerance and effectiveness of tVNS in patients suffering from moderate to severe GAD, CP and IBS in the context of a small-scale investigation performed in a private healthcare center.

\section{Materials and Methods}

\section{Participants}

This prospective study was conducted in agreement with the Declaration of Helsinki and was approved by the Institution Review Board of Kinesis Health Associates, Dartmouth, NS, Canada (ClinicalTrials.gov \#NCT03440255). Patients, men and women, naive of VNS treatment $(20<$ age $<65$ years) were recruited in our healthcare center through in situ advertisements, in 1-month interval prior starting the research. Subjects were eligible if they were presenting with either a main diagnosis of GAD according to The Diagnostic and Statistical Manual of Mental Disorders, 5th Edition - DSM$5^{99}$, CP (musculoskeletal-related disorders, neuropathic pain) or IBS according to the Rome IV Diagnostic Criteria for Irritable Bowel Syndrome ${ }^{100}$, meeting the scoring questionnaires standard for these disorders (moderate to severe score) and have exhibited symptoms for at least 6 months (Table 1). Participants were excluded if : (i) they were also diagnosed with pregnancy, concomitant severe neurological or medical diseases such as neoplasms in activity, neurodegenerative diseases, chronic infectious diseases uncompensated, concomitant severe psychiatric comorbidity, recent head trauma or concussion, severe alcoholism (ii) they were on drugs capable of affecting the
ANS (e.g., cholinergic, anticholinergic, antiarrhythmics, alpha or beta-blocking substance, cannabinoid drugs), (iii) pacemaker, active hearing aid such as cochlear implant, left ear wound and outer ear dermatologic affection, (iv), they had a past history of cervical vagotomy, neck chemotherapy or radiotherapy. After screening and in accordance with the inclusion and exclusion criteria, eligible participants provided written informed consent. The screening consisted of a detailed case history and a physical examination. Out of the 17 patients screened, 10 participants met the inclusion criteria and were enrolled in this stud and were allocated to one of the study's groups (GAD, CP or IBS) according to their diagnosed condition.

\section{tVNS treatment protocol}

Transcutaneous vagus nerve stimulation was performed by means of a standard lightweight and portable TENS stimulator (TU7000 Digital TENS device, RMP ltd, NS, Canada) to stimulate the left auricular branch of the VN. The stimulus generated a continuous asymmetric biphasic low-intensity square pulse. The patients were resting comfortably on a couch in a semi-supine position in a room at $21 \pm 2^{\circ} \mathrm{C}$. The stimulation site was cleaned up according to standard procedure and the conductive gel was applied on the electrodes ear clips to improve the conductivity. The electrode clips were respectively plugged in the cymba and the cavum conchae (Figure 1). The cathode and the anode were kept approximately $5 \mathrm{~mm}$ apart. The stimulation parameters, pulse frequency $(\mathrm{Hz})$ were chosen assuming that high frequencies impulses will stimulate more the afferent fibers of the VN to stimulate the CAN and HPA axis, and low frequencies impulses will act more on the efferent fibers to activate the CAP ${ }^{25,101}$. The pulse width (PW), expressed in $\mu \mathrm{s}$, ranged from $80 \mu$ s to $250 \mu \mathrm{s}$ as this range was demonstrated to elicit significant clinical effects. Indeed, tVNS showed to induce significant changes in cerebral activation patterns with shortmedium PW setting $(<250)$, showing marked deactivation

Table 1. Inclusion characteristics of the subjects

\begin{tabular}{|c|c|c|c|c|}
\hline Gender & Age & Main diagnosis & Duration of current episode (months) & Medication \\
\hline$f$ & 38 & IBS & 96 & Pro, Zop \\
\hline$f$ & 59 & IBS & 36 & Ami \\
\hline$m$ & 33 & IBS & 13 & Can, Pro \\
\hline$m$ & 37 & Chronic centralized pain & 14 & Bac, Can, Esc, Pan \\
\hline$m$ & 64 & Chronic arthritis & 48 & APO, Syn, Tra \\
\hline m & 40 & Chronic centralized pain & 8 & Na \\
\hline$f$ & 46 & Generalized anxiety & 48 & Hyd, Ami \\
\hline$f$ & 39 & Generalized anxiety & 48 & Par, Div \\
\hline$f$ & 43 & Generalized anxiety & 36 & Dul, Pre \\
\hline m & 38 & Generalized anxiety & Flu \\
\hline
\end{tabular}

demographic and diagnostic characteristics assessed at the screening visit during the baseline period (4 weeks). $f$, female; $m$, male; Pro, probiotics; Zop, zoplicone; Ami, amitriptyline; Can, cannbidiol oil; Bac, baclofen; Esc, Escitalopram; Pan, Pantapraxole; APO; apo-celocoxib; Syn, syntroid; Tra, Trandolapril; Nap, naproxen; Hyd, hydroclorothiazide; Par, paroxetine; Div, divalproex; Dul, duloxetine; Preg, pregabalin; Flu, fluoxetine 


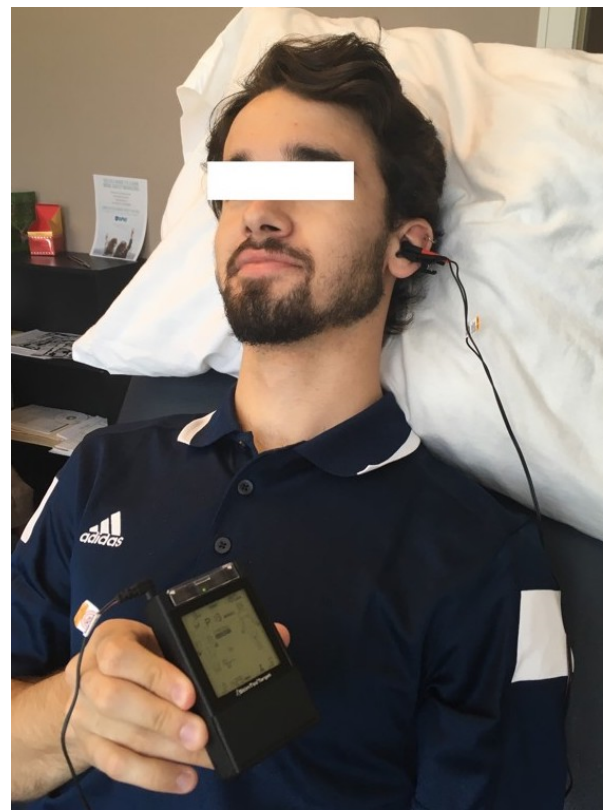

Figure 1: TENS device and emplacement of the electrodes for stimulation of the auricular branch of the vagus nerveVN in the concha.

of limbic and temporal brain areas in functional magnetic resonance imaging that suggests its application for psychiatric, mood-related disorders patients ${ }^{102-104}$. Pulse width $\geq 250$ were classically used in studies reporting significant VNS effects in various chronic pain syndromes (e.g., fibromyalgia and decreasing central sensitization in chronic pain, headaches), and in researches targeting inflammatory conditions of the digestive tract (e.g., enhancing gastroduodenal motility and reducing somatic pain sensitivity, and Inflammatory bowel disease) ${ }^{11,91,105}$. Although PW duration is intensity-dependant to cause sufficient nerve depolarization, i.e., short PW requires more intensity to elicit depolarization ${ }^{104}$, we estimated that low-intensity current $(<2 \mathrm{~mA})$ would elicit a clinical response at short $\mathrm{PW}(<150)$.

As VNS effectiveness is also frequency-dependent and frequency above $50 \mathrm{~Hz}$ was reported and confirmed to damage the vagus nerveVN ${ }^{106-108}$, the stimulation parameters were defined as follow: GAD: $20 \mathrm{~Hz}-80 \mu \mathrm{s}, \mathrm{CP}$ : $5 \mathrm{~Hz}-200 \mu \mathrm{s}$ and IBS: $3 \mathrm{~Hz}-250 \mu \mathrm{s}$. The intensity display $(\mathrm{mA})$ was not available on the device, therefore the stimulus intensity was individually adjusted to a level of perceptible fluttering and pleasant vibrating sensation just below the participant's pain threshold (pricking or unpleasant tingling sensation). That corresponds to a low intensity current $(0,8 \pm 0,3 \mathrm{~mA})$ (A-fibers), which, usually, represents the detection threshold in the cymba conchae ${ }^{17,109,110}$. Each stimulation session lasted 30 minutes.

\section{Study Overview}

This study was a 16-week, open label, non-randomized and not sham controlled, small-scale investigation. As no commonly approved protocol, for tVNS in the treatment of chronic GAD, IBS or CP was available in the literature, we chose arbitrarily to administer a low intensive, short term, treatment plan. The trial included ten visits for all participants: a visit during the 4-week screening time (baseline), eight stimulation sessions (twice a week for a duration of 4-week) and a final follow-up visit from day 90 to day 97. A maximum of three days separated the subsequent session (Figure 2). After written consent was obtained, eligible participants were informed about the experimental protocol and were asked to complete specific measure related to the group they were assigned in.

We measured the clinical improvement on GAD, CP, and IBS using three distinctive standard self-administrated questionnaires as primary endpoint. Measures were taken at baseline (BL), at the end of the treatment phase (ET = week 4) and at 2-month follow-up (FUp). At ET, all participants were also asked to fill in our own designed Safety and Tolerance questionnaire (supplement material). Clinical significance will be considered by a reduction in the score severity.

- Generalized anxiety disorder. The response effect of the tVNS on the severity of anxiety disorders

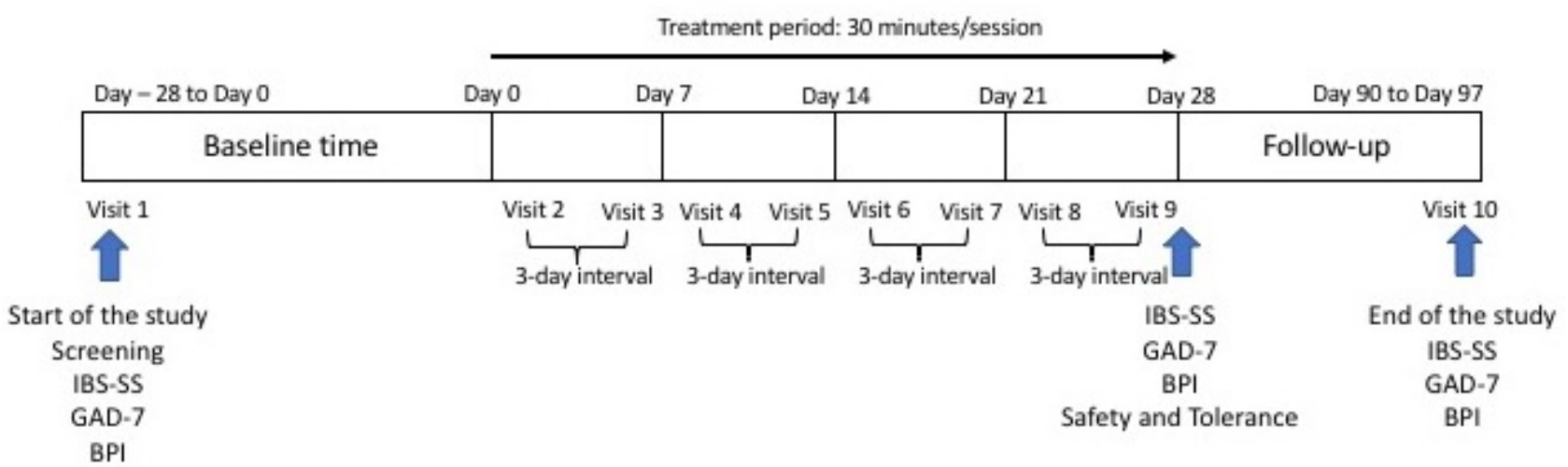

Figure 2: Study design. 
was measured by the 7 items Generalized Anxiety Disorder GAD-7 questionnaire with a total score ranging from 0 to 21 , where scoring scores of 5,10 and 15 represent cut-off points for mild, moderate and severe anxiety. Rating from 5-9 correlates with mild anxiety; 10-14, moderate anxiety and 15-21, severe anxiety ${ }^{111}$.

- Pain severity. The response effect of the tVNS on the severity of pain (pain perception only) was measured by the 9 items Brief Pain Inventory (BPI) questionnaire (Short Form); left end point « 0 » (no pain), right end point « $10 »$ (worst pain), ratings from 1-4, mild pain; 5-6, moderate pain, and 7-10, severe pain with cut-off points being 4 and 6 . Scoring pain severity is the mean of the total pain score out of $10^{112}$.

- Irritable bowel syndrome. The response effect of the tVNS on the severity of irritable bowel syndrome measured by the Irritable Bowel Syndrome Severity Scoring System (IBS-SSS), where the maximum achievable score is 500 . Mild, moderate and severe categories were indicated by scores of 75 to 175 , 175 to 300 and > 300 respectively, with cut-off points being 175 and $300^{113}$.

- Safety and Tolerance. The response effect of the tVNS on safety and tolerance was screened by a simple self-administrated 9-closed questions questionnaire that reports adverse events (AEs). This questionnaire was designed for the purpose of the study (supplement material). Adverse events were defined as symptoms experienced during or after the stimulation, as a consequence from it. The first 6 items are related to AEs experienced during the stimulation, i.e. alterations of pain, headaches, throat ache, itching, nausea and voice (left end point « not at all », right end point « every time »). Item 7 and 8 are related to the presence of temporary and permanent AEs ("yes" or "no") in between sessions. Item 9 is related to the overall satisfaction at the end of the treatment phase (left end point « not at all », right end point « very satisfied »).

\section{Statistical analysis}

We performed a power analysis to estimate the power we would reach for our low sample size, in each group. The power calculation was performed using $G^{*}$ Power, version 3.1.9.4 for Windows. A one-way within-subjects analysis of variance was performed to compare the effects of tVNS on the severity of GAD, IBS and CP, from baseline to the end of treatment and 2month follow-up. A Bonferroni adjustment was conducted to protect from Type I error. A simple linear regression analysis was used to explore the relationship between the effect of time, at the end of tVNS and at follow-up on GAD, IBS and $\mathrm{CP}$ intensity. Finally, a Pearson correlation was performed to examine the relationships between GAD, IBS and CP and the satisfaction level reported at the end of the treatment. The analysis was performed using the IBM Statistical Package for Social Science (SPSS) version 24 for Windows, and significance level was defined as $p<0.05$.

\section{Results}

Out of 10 participants included in the study (five women, five men; mean age \pm SD years: $45.18 \pm 10.74$ ) one patient dropped out from the GAD group at follow-up due to scheduling conflicts.

The power was low (0.38), considering a total sample size of 3 (per group), an effect size of 0.65 and Partial $\eta^{2}=$ 0.30 .

Four patients were included in the GAD group. The mean \pm SD GAD-7 score varied from $17.75 \pm 1.49$ on inclusion to $11.25 \pm 3.06$ (4-week) and to $11.66 \pm 2.43$ at 2 months follow-up. Three patients were included in the CP group. The mean \pm SD BPI pain severity score ranged from $5.25 \pm 0.66$ on inclusion to $4.83 \pm 0.50$ ( 4 -week) and to 5.41 \pm 1.34 at follow-u. The IBS group included three patients. The mean \pm SD IBS-SSS score varied from $350 \pm 64.29$ on inclusion to $299.33 \pm 94.06$ (4-week) and to $252.33 \pm$ 85.04 at follow up (Table 3). At 2-month follow-up 50\% of patients from the GAD group improved their GAD-7 score; $75 \%$ of the IBS group improved their IBS-SSS score while no improvement was achieved in the CP group.

The score of generalized anxiety disorder, chronic pain and Irritable bowel syndrome at the end of treatment and at 2-month follow-up, compared to pre-treatment, are summarized below (Table 2).

We present, in Table 3, the data of each participant's score for each group. Assuming that our data are normally distributed, we conducted a one-way within-subjects ANOVA to compare the effect of time of tVNS on severity of GAD, IBS and CP, at the end of treatment and at 2month follow-up. In term of the effect of time we didn't reach significance difference for all the group tested, after treatment and at follow-up. Indeed, for the anxiety group, Wilks' Lambda $=0.158, \mathrm{~F}(2,2)=5.31, p=0.158$. For the IBS

Table 2. Clinical outcome (pre, post-treatment, follow-up) according to disorder and stimulation parameters.

\begin{tabular}{|c|c|c|c|c|c|c|}
\hline & $\mathbf{n}$ & GAD $(20 \mathrm{~Hz}-80 \mu \mathrm{s})$ & $\mathbf{n}$ & 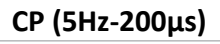 & $\mathbf{n}$ & IBS (3Hz-250 $\mu \mathrm{s})$ \\
\hline Pre-treatment & 4 & $17.75 \pm 1.49$ & 3 & $5.25 \pm 0.66$ & 3 & $350 \pm 64.29$ \\
\hline Post-treatment & 4 & $11.25 \pm 3.06$ & 3 & $4.83 \pm 0.50$ & 3 & $299.33 \pm 94.06$ \\
\hline Follow-up & 3 & $11.66 \pm 2.43$ & 3 & $5.41 \pm 1.34$ & 3 & $252.33 \pm 85.04$ \\
\hline
\end{tabular}


Table 3. Participants' scores for anxiety, irritable syndrome and chronic pain at baseline, after treatment and at follow-up.

\begin{tabular}{|c|c|c|c|c|c|c|c|c|}
\hline GAD_BL & GAD_ET & GAD_FUp & IBS_BL & IBS_ET & IBS_FUp & CP_BL & CP_ET & CP_FUp \\
\hline 14.00 & 5.00 & 7.00 & 470.00 & 415.00 & 390.00 & 5.50 & 4.75 & 7.00 \\
\hline 19.00 & 17.00 & 15.00 & 330.00 & 370.00 & 270.00 & 4.00 & 4.00 & 2.75 \\
\hline 17.00 & 16.00 & $99.00 *$ & 250.00 & 113.00 & 97.00 & 6.25 & 5.75 \\
\hline 21.00 & 11.75 & 13.00 & & & & & & \\
\hline
\end{tabular}

BL, baseline; ET, end of treatment, FUp, follow-up. $\left({ }^{*}\right)$ : in the GAD-FUp group, 99.00 represents a missed value (for computation only) due to the dropped-out patient.

group, Wilks' Lambda $=0.37, \mathrm{~F}(2,1)=13.11, p=0.192$, and for the CP group, Wilks' Lambda $=0.13, \mathrm{~F}(2,1)=37,56, p$ $=0.115$.

A bivariate regression was conducted to examine how well effect of time after tVNS could predict level score on GD, IBS and CP. A scatterplot showed that the relationship between time and GAD and IBS was negative and linear and did not reveal any bivariate outliers. On the other hands, the relationship between time and CP was positive. There was no statistically significant correlation between effect of time after tVNS and GAD, $r(9)=0,51, p=0,1$. The coefficient of determination for this model $r^{2}$ was 0,26 , that correspond to an medium relationship ${ }^{114}$. We found the same observation for the IBS group, $r(8)=0,32, p=0,39$, with a smaller relationship, $r^{2}=0,1$. No statistically significance correlation was found between effect of time and CP, $r(7)=0,26, p=0,94$ and $r^{2}=0,003$ that showed no relationship for this model. The general trend effects of tVNS over time, from baseline to follow-up, is presented in Figure 3.

Transcutaneous vagus nerve stimulation was well tolerated. No serious or permanent AEs wasere reported at the end of the treatment (experimental phase). Although temporary symptoms were accounted for $56 \%$ of the total number of cases, most frequently reported AEs lasted only during the stimulation sessions, rarely in-between the sessions: pain along the homolateral upper side of the neck $(19,44 \%)$, headaches $(8,33 \%)$, itching at the stimulation site and nausea (5,56\%) (Table 4).

The mean of $\Sigma$ AEs accounted for $\mathrm{m}=6.5 \%$. The safety of tVNS was calculated as follow: $100 \%-6.5 \%=93.50 \%$.
Overall, $56 \%$ of the participants were satisfied with the outcome of the tVNS at the end of the treatment phase.

A Pearson product-moment correlation coefficient was computed to examine the relationships between subjects 'satisfaction after tVNS at ET-time and their score level of GAD, IBS and CP. There was a strong negative correlation between satisfaction and anxiety, $r(2)=-0.98, p<0.05$. Although it seems to have a strong correlation between satisfaction and IBS, we cannot conclude that it exists, $r$ (1) $=-0.99, p>0.05$. No correlation was found between satisfaction and pain level, $r(1)=-0.08, p>0.05$. Scatterplots summarize the results in (Figure 4). Increase in satisfaction was only correlated with decrease in rating of anxiety.

\section{Discussion}

To the best of our knowledge, this is the first study exploring the safety, tolerance and clinical effects of tVNS, on general anxiety disorders, irritable bowel syndrome and chronic pain, in a private healthcare center. Incorporating nVNS therapy into private practice was poorly explored. One study assessed this feasibility and it was suggested that patient management under VNS could be performed in a general neurology practice to patients suffering from drug-resistant epilepsy ${ }^{115}$. It was therefore interesting to explore the clinical benefits and feasibility of noninvasive vagus nerve stimulation that a private healthcare center could offer as it was demonstrated that tVNS was a cheaper alternative or support to conventional treatment and that patients reported to prefer using tVNS than standard of care ${ }^{116,117}$. Before discussing the results, several limitations of our study have to be considered. First, this

Table 4. Adverse events experienced by tVNS during the treatment phase (Day-0 to Day-28).

\begin{tabular}{|c|c|c|c|c|c|}
\hline Pain & Itching & nausea & throat ache & Voice & Headaches \\
\hline 0 & 0 & 0 & 0 & 0 & 25 \\
\hline 25 & 0 & 25 & 0 & 0 & 0 \\
\hline 0 & 25 & 0 & 0 & 0 & 25 \\
\hline 25 & 0 & 0 & 0 & 0 & 0 \\
\hline 25 & 0 & 0 & 0 & 0 & 0 \\
\hline 0 & 0 & 25 & 0 & 0 & 0 \\
\hline 25 & 0 & 0 & 0 & 0 & 0 \\
\hline 25 & 0 & 0 & 0 & 0 & 25 \\
\hline $19.44 \pm 16.67$ & $5.56 \pm 11.02$ & $5.56 \pm 11.02$ & 0 & 0 & $8.33 \pm 12.50$ \\
\hline
\end{tabular}

data are expressed as (\%) mean \pm SD. 


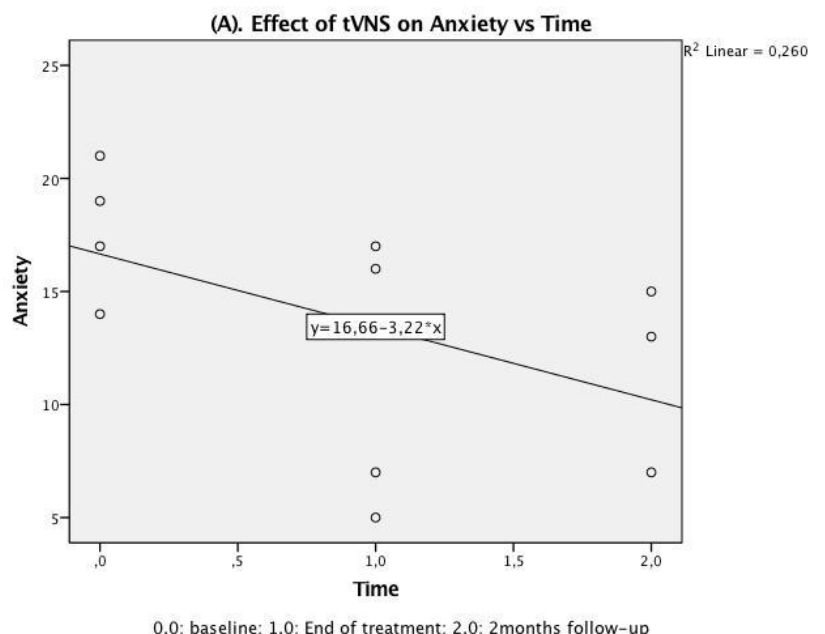

0,0: baseline; 1,0: End of treatment; 2,0: 2months follow-up

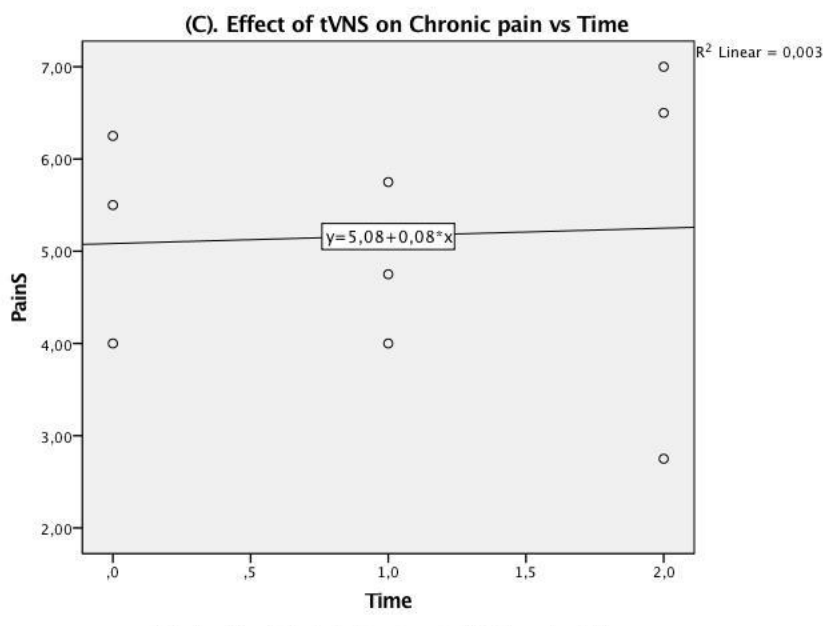

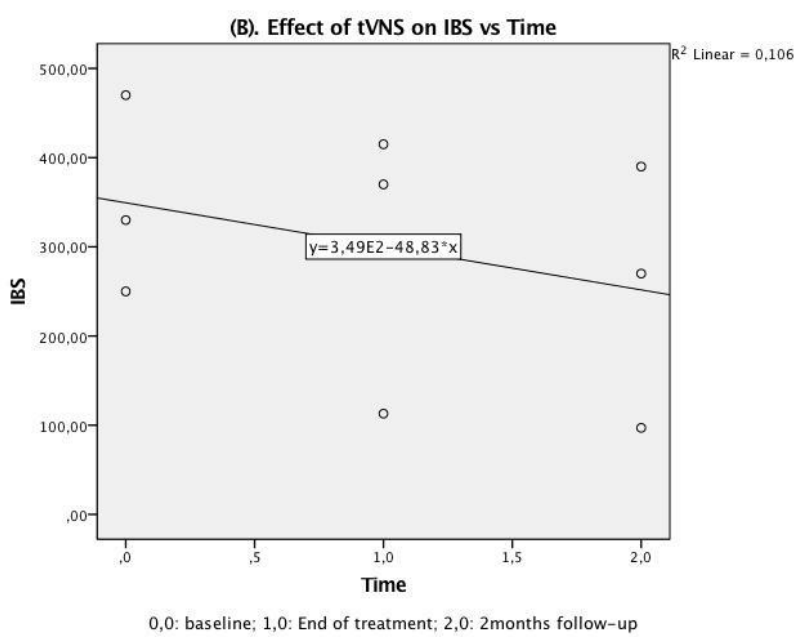

0,0: baseline; 1,0: End of treatment; 2,0: 2months follow-up

0,0: baseline; 1,0: End of treatment; 2,0: 2months follow-up

Figure 3: Scatterplot for trend effects of transcutaneous vagus nerve stimulation over time on condition from baseline to 2-month followup on: (A) Generalized anxiety disorders - GAD-7 questionnaire, (B) irritable bowel syndrome - IBS-SSS questionnaire and (C) chronic pain - BPI (short form) questionnaire.

small-scale investigation included a small sample size in each group (GD, CP, , and IBS). In this case, our repeated measures design didn't allow us to conduct a reliable and statistically significant analysis. Indeed, a minimum sample size of 6 participants per group, with 3 measurements at a significance level of 0.05 , would have been required to reach statistical power of 0.80 with an effect size of 0.65 and a $\eta_{p}^{2}=0.30$. To test this assumption, we resampled our data for GAD (doubling them, i.e.,8 participants) and IBS (doubling them, i.e., 6 participants) and tripled our data for CP ( 9 subjects). We then reconducted all our statistical analysis and found statistical significance in our withinsubject repeated measures, regression and correlation analysis (results not shown). This test pointed out that our sample size for each group was indeed insufficient to achieve statistical significance. Second, the participants suffered from three different disorders that would have been better considered in separate studies as responses to vagal modulation is known to vary considerably in time and intensity according to the targeted pathology ${ }^{10,34,41,91,118}$. Third, only subjective measures were analyzed and placebo effect was impossible to exclude. By consequence, we were unable to demonstrate statistically significant effects of tVNS on GAD, IBS and CP at the end of treatment and at follow-up.

However, we observed that tVNS is likely safe and was well tolerated with more than $50 \%$ satisfaction reported. Moreover, we found moderate clinical effects of tVNS on anxiety and IBS over time. Interestingly, we noticed a significant strong negative correlation between satisfaction and anxiety level compared to the other groups where no significant correlation was reached. Indeed, although the GAD group showed a medium (non-significant) relationship between effect of time after tVNS $(p=0,1)$, it was the strongest relationship found compared to the other groups, e.g., $p=0.39$ and $p=0.94$ for the IBS and 

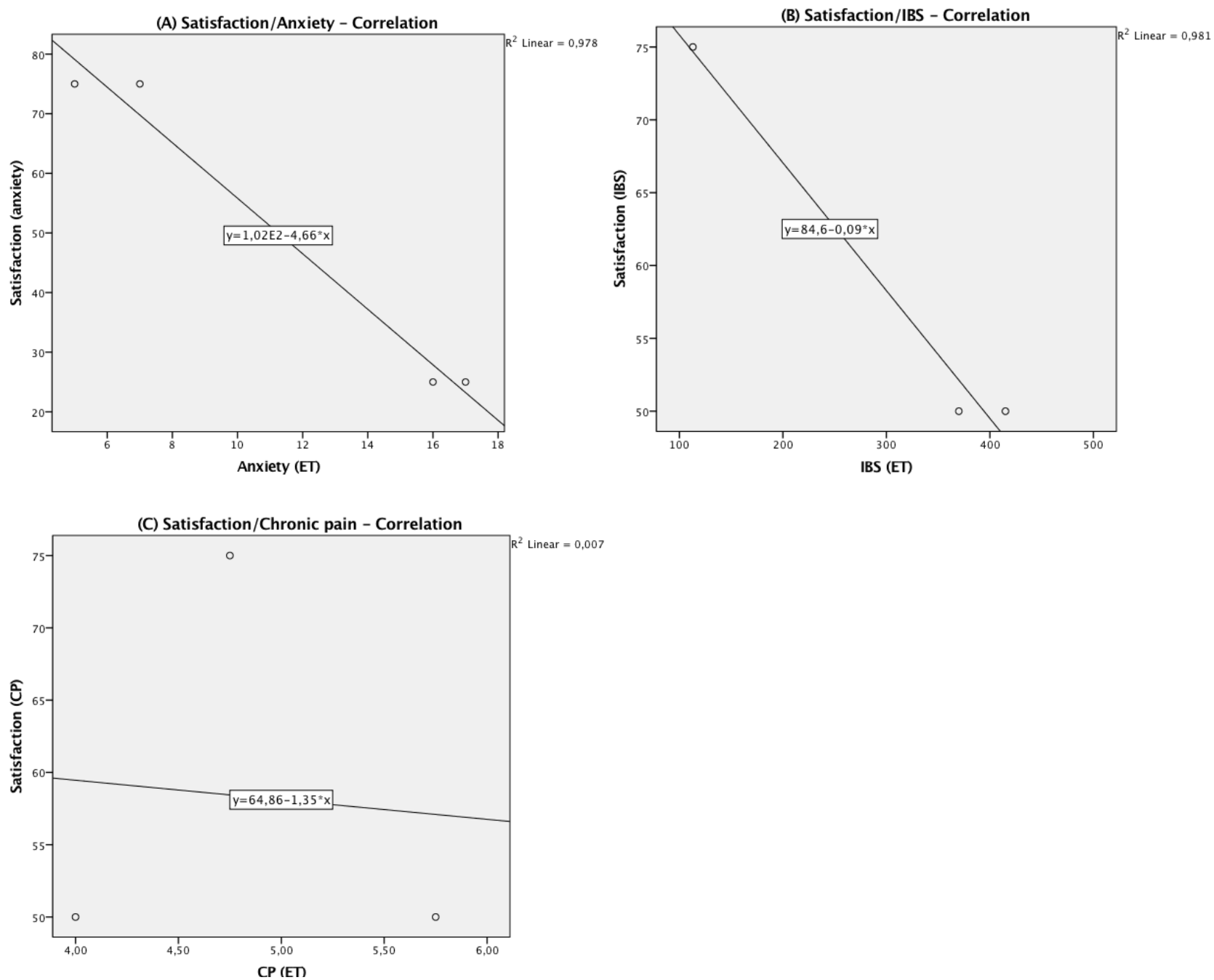

Figure 4: Scatterplot for correlation between satisfaction and the measured outcome at the end of treatment: (A) generalized anxiety disorders, (B) irritable bowel syndrome and (C) chronic pain - BPI.

CP group, respectively. These observations might suggest that the GAD group reacted better clinically then the other groups, although this should be read carefully.

Unfortunately, we were not able to yield a clinical effect on chronic pain. Given the results, one could argue the following key questions: first, why tVNS seems to clinically improved patients suffering from GAD and IBS after 8 stimulations sessions that still lightly persisted at 2-months follow-up as shown by the trend line (Fig. 3A, Fig. 3B) but not to patients suffering from CP (Fig. 3C)? Despite the reduced number of participants in all groups, could the settings defined for each group have influenced the outcome of the groups? Second, how could we effectively and easily monitor the benefits from tVNS therapy in a private healthcare out-patient center to support its use, i.e., is there any objective, non-invasive, non-expensive means that would help to assess the real physiological impact of such a therapy? To answer the first question, we will discuss the impact of VNS parameters on the vagal afferent and efferent fibers for these affections and how it might have interfered with our results.

Unfortunately, we failed to confirm the encouraging results of studies that reported significant positive effects of VNS or tVNS on pain modulation ${ }^{91}$. Indeed, regardless our too small sample size in the $\mathrm{CP}$ group, one possible reason that could explain our failure in reducing pain perception as per our study protocol is that implanted VNS (chronic stimulation) may exert its effect with some latency as it was shown in the treatment of epilepsy, Crohn's disease or patients suffering from fibromyalgia with effectiveness gained over time ${ }^{35,67,119}$. In another study exploring the effect of implantable VNS on chronic migraine, partial or complete relief were obtained in about 1 month $^{120}$. Therefore, our acute tVNS design might have been insufficient to reach clinical improvement in chronic pain. Now, if the antinociceptive effects of tVNS was demonstrated in healthy volunteers and in patients, it was also shown that chronic pain is often associated with comorbidities like anxiety, 
mood disorders or depression and IBS. Indeed, it was established that the presence of multiple anxiety, mood or depressive disorders were significantly associated with disability from pain as it was seen in up to $35 \%$ of painrelated patients in primary care clinics ${ }^{94,121-123}$. As the participants in the CP group were not specifically screened for comorbidities that might potentially have influenced the outcome, e.g., the poor effect of the tVNS that we have noticed in this group. Furthermore, another possible reason that may explain the results reached in the CP group could be linked with possible non-specific symptoms that may have suggested central sensitization in some of the participants. In that case, the patient management is more complex and the stimulation setting probably different, i.e., higher frequency would have been preferably chosen ${ }^{124,125}$. Indeed, as we measured only the pain perception we were not able to identify its underlying mechanisms, even if the mean pain level reported before and after the tVNS was moderate ${ }^{126}$. Analyzing established measures that would have incorporated the four domains of pain, i.e. nociception, neuropathy, inflammation, and central sensitization would probably help us to better understand the non-response to tVNS after 8 stimulation sessions in this group ${ }^{127}$. VNS stimulation parameters are another significant factor to think about to elicit the physiological reactions that will produce a therapeutic effect based on targeting the afferent or efferent vagus nerve fibers. Several studies have shown that the response to VNS is frequency/dose-dependent. For instance, low-frequency stimulation $(5 \mathrm{~Hz})$ that stimulates the efferent fibers induced the activation of the CAP in animal models ${ }^{78,101,128,129}$ but didn't show significant brain activation compared to a higher frequency in depression and epileptic patients ${ }^{130-132}$. However, one survey showed short-term brain activation induced by low-frequency (8 $\mathrm{Hz}$ ) tVNS in healthy subjects ${ }^{102}$, and in a randomized doubleblind trial,Straube etal. showed evidence of the effectiveness of tVNS for chronic migraine on $1 \mathrm{~Hz}$ stimulation ${ }^{133}$. It was indeed previously demonstrated in an animal model of endotoxemia that VNS can produce its anti-inflammatory effect at very low-frequency $(1 \mathrm{~Hz})^{78}$. Furthermore, studies in humans demonstrated and confirmed that the activation of the CAP showed similar effects by activation of the vagal efferent fibers at low-frequencies $(5 \mathrm{~Hz}-10 \mathrm{~Hz})^{67,129,134}$. We set the frequencies parameters for our sample suffering from GAD at $20 \mathrm{~Hz}$ which are a standard for stimulating the vagal afferents. In agreement with the above-mentioned evidence, we chose as well low frequencies to stimulate the CAP for our IBS and CP samples respectively at $3 \mathrm{~Hz}$ and $5 \mathrm{~Hz}$. The PW chosen for the GAD group fell within the spectrum of accepted range that showed significant central projections of the vagus nerveVN and clinical response. Indeed several studies suggested that central projections of the VN are affected by a PW ranging from $20 \mu$ s to 500 $\mu \mathrm{s}^{13,18,59,102,135}$. For instance, Fang et al reported modulation of the default mode network after tVNS with short PWpulse width $(<100 \mu \mathrm{s})^{29}$. fMRI studies conducted by Kraus et al showed changes in BOLD signal in the limbic system and the brain stem with mood enhancing effect using short PW-pulse width $(20 \mu \mathrm{s})$ and low-frequency stimulation parameters of the AVBN $(8 \mathrm{~Hz})^{102,103}$. The settings for the IBS group ranged in the VNS parameters aligned with those from previous studies that reported the activation of the CAP, i.e. $10 \mathrm{~Hz}$ and lower. Our clinical results thus confirmed that $3 \mathrm{~Hz}-250 \mu$ s may be enough to target the CAP efficiently and obtain a clinical response. While we had patients diagnosed with chronic central pain, we might have preferably chosen higher frequencies, i.e., $20 \mathrm{~Hz}$ or 25 $\mathrm{Hz}$, as commonly chosen to stimulate the afferent fibers of the VN vagus, therefore targeting preferably the brain pain modulation circuitry instead of the CAP. In our study, we defined low-frequency stimulation $(5 \mathrm{~Hz}-200 \mu \mathrm{s})$ for the CP group. The poor effects of tVNS noticed for these patients might also have been associated with damaged VN. Indeed, it was showed that patients suffering from non resolving inflammation develop impairs vagus nerveVN signaling, therefore accelerating the progression of inflammation and preventing its resolution ${ }^{136}$. Interestingly, this could explain as well why tVNS failed in its antinociceptive effect, even at low-frequency stimulation. Bonaz and its team recently confirmed the results obtained by Borovika et al., and others, that it was possible to activate vagal afferents in the brain using low-frequency stimulation, i.e., modulation of the CAN and/or the HPA axis ${ }^{78,130,137}$. Together with the HPA axis, the periaqueductal gray matter (part of the CAN) and in close connection with the nucleus raphe dorsalis, that are major targets in the activation of the ascending antinociceptive pathway.

Our second question pointed out how we could easily monitor the benefits from tVNS therapy, e.g., the sympathovagal balance. Screening only subjective measurements of the effectiveness of tVNS for patients might not be enough to justify its use in primary care as safety couldn't be assumed without measuring the treatment's physiological effects. Altogether, subjective and objective measurement, along the course of this therapy, may yield better patient management in private healthcare facilities if powerful, controlled, blinded studies may support its use. Heart rate (HR), respiratory rate (RR) and heart rate variability (HRV) analysis are possible and easy methods to assess autonomic regulation, therefore they could potentially be used to gauge the physiological effect of vagus nerve stimulationVNS. However, HRV analysis, i.e., the beatto-beat variation in either heart HR or heart period (R-R interval) ${ }^{138}$, based on short-term ECG, would be more interesting to estimate vagal modulation as it does include separated information about the autonomic function, that cannot be obtained from HR measures only, i.e., vagal modulation distinct from sympathetic influence: heart 
rate increasing by sympathetic action and decreasing by parasympathetic activity ${ }^{139}$. The same limitations will apply when considering RR alone, as respiration is both influenced by the sympathetic and the parasympathetic input as inhalation is mediated by the former and exhalation by the later. The coupling of HR and respiratory cycle is called respiratory sinus arrhythmia (RSA). RSA, which is the variation of the heart while breathing, i.e., HR increases during inspiration and decreases during expiration, corresponds to HRV in synchronicity with respiration and is vagally mediated if the breathing cycle remains between 9 and $24 \mathrm{cycle} / \mathrm{min}^{138,140,141}$. Therefore, HRV seems to be a biomarker of choice to appraise vagal tone index, e.g., makers from the time-domain, frequencydomain or non-linear analysis. The root mean square of successive R-R interval differences (RMSSD) and the percentage of successive R-R intervals that differ by more than $50 \mathrm{~ms}$ from the preceding one (pNN50) are commonly used markers of vagal tone that are retrieved from the time-domain measures. The frequency-domain analysis and its high frequency power component $(0.15-0.4 \mathrm{~Hz})$ in normalized unit, and non-linear markers, e.g., the Poincaré plot standard deviation perpendicular to the line of identity (SD1) that reflects short-term HRV, may be used to assess the vagal tone as well ${ }^{142,143}$. Furthermore, It was demonstrated that mood disorders like depression and anxiety, gastrointestinal inflammation and irritation and chronic pain syndromes are correlated with a sympathovagal imbalance in favour of an over activation of the sympathetic and a decrease of vagal activity as measured by HRV $^{144-149}$. It was showed that tVNS effect, as estimated by HRV, induces a shift in cardiac autonomic function toward parasympathetic predominance. Moreover, auricular electroacupuncture increases RSA, mediated by the vagus nerveVN ${ }^{150,151}$. Following the recommendations for heart rate variability assessment ${ }^{152}$, HRV would be a feasible, reliable and non-invasive method to assess sympathovagal balance in eligible patients for tVNS before and along with the treatment plan.

\section{Conclusion}

The present original study conducted in a private healthcare center didn't show significant clinical improvement after acute tVNS for patients suffering from GAD, IBS and CP although a negative and linear, moderate and small relationship was found as the relationship between the effect of time on GAD and IBS. The explanation for our poor results and mitigated clinical improvement may reside in the fact that: (i) the small sample size in each group didn't allow us to statistically assess the effectiveness of tVNS therapy, (ii) the stimulation settings, frequency and pulse width might have not been optimally chosen, especially for the CP group. Our assessment was based only on subjective measurements but it gave us an idea of the possible clinical effects of tVNS for patients suffering from
GAD, IBS and CP, as it was already supported by available evidence. We observed that tVNS is likely safe and was well tolerated The supposed contribution of the placebo effect cannot be concluded. Future research may wish to delve deeper into these issues and examine them in more detail while addressing our study limitations to support the feasibility of transcutaneous vagus nerve stimulation in private healthcare center.

\section{Author Information}

Pascal J.D. Grolaux, Department of Osteopathy, Kinesis Health Associates, Dartmouth, Nova Scotia, Canada. Pascal J.D. Grolaux designed, conducted the study, including recruitment, investigations, data analysis, reviewed literature and wrote this manuscript.

\section{Acknowledgments}

The author thanks Christene Missener who helped in the clinical phase and Alex Mader who made data collection and pre-analysis.

\section{References}

1. Verlinden TJ, Rijkers K, Hoogland G, et al. Morphology of the human cervical vagus nerve: implications for vagus nerve stimulation treatment. Acta Neurol Scand. 2016; 133(3): 173-82.

2. Cheng G, Zhou $\mathrm{X}, \mathrm{Qu}$ J, et al. Central vagal sensory and motor connections: human embryonic and fetal development. Auton Neurosci. 2004; 114(1-2): 83-96.

3. Tracey KJ. Reflex control of immunity. Nat Rev Immunol. 2009; 9(6): 418-28.

4. Chavan SS, Tracey KJ. Essential Neuroscience in Immunology. J Immunol. 2017; 198(9): 3389-97.

5. Jonge W. The Gut's Little Brain in Control of Intestinal Immunity. Isrn Gastroenterol. 2013; 1-17.

6. Grimm S, Bajbouj M. Efficacy of vagus nerve stimulation in the treatment of depression. Expert Rev Neurother. 2010; 10(1): 87-92.

7. Beekwilder JP, Beems T. Overview of the clinical applications of vagus nerve stimulation. J Clin Neurophysiol. 2010; 27(2): 130-8.

8. Lulic D, Ahmadian A, Baaj AA, et al. Vagus nerve stimulation. Neurosurg Focus. 2009; 27(3): E5

9. Attenello F, Amar AP, Liu C, et al. Theoretical Basis of Vagus Nerve Stimulation. Prog Neurol Surg. 2015; 29: 20-8.

10. Johnson RL, Wilson CG. A review of vagus nerve stimulation as a therapeutic intervention. J Inflamm Res. 2018; 11: 203-13.

11. Bonaz B, Sinniger V, Pellissier S. Vagus nerve stimulation: a new promising therapeutic tool in inflammatory bowel disease. J Intern Med. 2017; 282(1): 46-63.

12. Dietrich S, Smith J, Scherzinger C, et al. [A novel transcutaneous vagus nerve stimulation leads to brainstem and cerebral activations measured by functional MRI]. Biomed Tech. 2008; 53(3): 104-11.

13. Frangos E, Ellrich J, Komisaruk BR. Non-invasive Access to the Vagus Nerve Central Projections via Electrical Stimulation of the External Ear: fMRI Evidence in Humans. Brain Stimul. 2015; 8(3): 624-36.

14. Mercante B, Deriu F, Rangon CM. Auricular Neuromodulation: The Emerging Concept beyond the Stimulation of Vagus and Trigeminal Nerves. Med. 2018; 5(1). 
15. Peuker ET, Filler TJ. The nerve supply of the human auricle. Clin Anat 2002; 15(1): 35-7.

16. Bermejo P, Lopez M, Larraya I, et al. Innervation of the Human Cavum Conchae and Auditory Canal: Anatomical Basis for Transcutaneous Auricular Nerve Stimulation. Biomed Res Int. 2017; 1-10.

17. Ellrich J. Transcutaneous Vagus Nerve Stimulation. Eur Neurol Rev. 2011; 6(4): 254-256

18. Yakunina N, Kim SS, Nam EC. Optimization of Transcutaneous Vagus Nerve Stimulation Using Functional MRI. Neuromodulation. 2017 20(3): 290-300

19. Safi S, Ellrich J, Neuhuber W. Myelinated Axons in the Auricular Branch of the Human Vagus Nerve. Anat Rec. 2016; 299(9): 1184-91.

20. Krahl SE, Senanayake SS, Handforth A. Destruction of peripheral C-fibers does not alter subsequent vagus nerve stimulation-induced seizure suppression in rats. Epilepsia. 2001; 42(5): 586-9.

21. Nomura S, Mizuno N. Central distribution of primary afferent fibers in the Arnold's nerve (the auricular branch of the vagus nerve): a transganglionic HRP study in the cat. Brain Res. 1984; 292(2): 199205.

22. Badran BW, Dowdle LT, Mithoefer OJ, et al. Neurophysiologic effects of transcutaneous auricular vagus nerve stimulation (taVNS) via electrical stimulation of the tragus: A concurrent taVNS/fMRI study and review. Brain Stimul. 2017; 11(3): 492-500.

23. Benarroch EE. The central autonomic network: functional organization, dysfunction, and perspective. Mayo Clin Proc. 1993; 68(10): 988-1001.

24. Andersson U, Tracey KJ. Neural reflexes in inflammation and immunity. J Exp Med. 2012; 209(6): 1057-68.

25. Bonaz B, Sinniger V, Pellissier S. Anti-inflammatory properties of the vagus nerve: potential therapeutic implications of vagus nerve stimulation Blackwell Publishing Ltd. J Physiol. 2016 Oct 15; 594(20): 5781-5790.

26. Garamendi-Ruiz I, Gomez-Esteban JC. Cardiovascular autonomic effects of vagus nerve stimulation. Clin Auton Res. 2017.

27. Chen SP, Ay I, de Morais AL, et al. Vagus nerve stimulation inhibits cortical spreading depression. Pain. 2016; 157(4): 797-805.

28. Daban C, Martinez-Aran A, Cruz N, et al. Safety and efficacy of Vagus Nerve Stimulation in treatment-resistant depression. A systematic review. J Affect Disord. 2008; 110(1-2): 1-15

29. Fang J, Rong P, Hong Y, et al. Transcutaneous Vagus Nerve Stimulation Modulates Default Mode Network in Major Depressive Disorder. Biol Psychiatry. 2016; 79(4): 266-73.

30. Hein E, Nowak M, Kiess O, et al. Auricular transcutaneous electrica nerve stimulation in depressed patients: a randomized controlled pilot study. J Neural Transm. 2013; 120(5): 821-7.

31. Marshall R, Taylor I, Lahr C, et al. Bioelectrical Stimulation for the Reduction of Inflammation in Inflammatory Bowel Disease. Clin Med Insights Gastroenterol. 2015; 8: 55-9.

32. Napadow V, Edwards RR, Cahalan CM, et al. Evoked pain analgesia in chronic pelvic pain patients using respiratory-gated auricular vagal afferent nerve stimulation. Pain Med. 2012; 13(6): 777-89.

33. Ventureyra EC. Transcutaneous vagus nerve stimulation for partial onse seizure therapy. A new concept. Child's Nerv Syst. 2000; 16(2): 101-2.

34. Yu SY, Zhao JJ, Luo M, et al. Transcutaneous Auricular Vagus Nerve Stimulation: A Novel Non-InvasiveNon-Pharmacologic Treatment for Major Depressive Disorder. J Depress Anxiety. 2016; 5(3).

35. Lange G, Janal MN, Maniker A, et al. Safety and efficacy of vagus nerve stimulation in fibromyalgia: a phase I/II proof of concept trial. Pain Med. 2011; 12(9): 1406-13.
36. Kong J, Fang J, Park J, et al. Treating Depression with Transcutaneous Auricular Vagus Nerve Stimulation: State of the Art and Future Perspectives. Front Psychiatry. 2018; 9: 20.

37. Katzman MA, Bleau P, Blier P, et al. Canadian clinical practice guidelines for the management of anxiety, posttraumatic stress and obsessivecompulsive disorders. BMC Psychiatry. 2014; 14 Suppl 1: S1.

38. Kessler RC, Angermeyer M, Anthony JC, et al. Lifetime prevalence and age-of-onset distributions of mental disorders in the World Health Organization's World Mental Health Survey Initiative. World Psychiatry. 2007; 6(3): 168-76.

39. Somers JM, Goldner EM, Waraich $\mathrm{P}$, et al. Prevalence and incidence studies of anxiety disorders: a systematic review of the literature. Can J Psychiatry. 2006; 51(2): 100-13.

40. Definitions and Diagnostic Criteria for GAD [Internet]. Available from: www.ADAA.org

41. George MS, Ward Jr. HE, Ninan PT, et al. A pilot study of vagus nerve stimulation (VNS) for treatment-resistant anxiety disorders. Brain Stimul. 2008; 1(2): 112-21.

42. Zabara J. Inhibition of experimental seizures in canines by repetitive vagal stimulation. Epilepsia. 1992; 33(6): 1005-12.

43. George MS, Rush AJ, Sackeim HA, et al. Vagus nerve stimulation (VNS) utility in neuropsychiatric disorders. Int J Neuropsychopharmacol. 2003; 6(1): 73-83.

44. Goodnick PJ, Rush AJ, George MS, et al. Vagus nerve stimulation in depression. Expert Opin Pharmacother. 2001; 2(7): 1061-3.

45. Sundman E, Olofsson PS. Neural control of the immune system. Adv Physiol Educ. 2014; 38(2): 135-9.

46. Davison K. Autoimmunity in psychiatry. Br J Psychiatry. 2012; 200(5) 353-5.

47. Vogelzangs N, Beekman AT, de Jonge P, et al. Anxiety disorders and inflammation in a large adult cohort. Transl Psychiatry. 2013; 3: e249.

48. Breit S, Kupferberg A, Rogler G, et al. Vagus Nerve as Modulator of the Brain-Gut Axis in Psychiatric and Inflammatory Disorders. Front Psychiatry. 2018; 9: 44

49. Thayer JF, Sternberg EM. Neural aspects of immunomodulation: focus on the vagus nerve. Brain Behav Immun. 2010; 24(8): 1223-8.

50. Michopoulos V, Powers A, Gillespie CF, et al. Inflammation in Fear- and Anxiety-Based Disorders: PTSD, GAD, and Beyond. Neuropsychopharmacology. 2017; 42(1): 254-70.

51. Zhao YX, He W, Jing XH, et al. Transcutaneous auricular vagus nerve stimulation protects endotoxemic rat from lipopolysaccharideinduced inflammation. Evidence-Based Complement Altern Med. 2012; 2012: 627023

52. De Couck M, Mravec B, Gidron Y. You may need the vagus nerve to understand pathophysiology and to treat diseases. Clin Sci (London, Engl 1979). 2012; 122(7): 323-8.

53. Felger JC. Imaging the role of inflammation in mood and anxietyrelated disorders. Curr Neuropharmacol. 2017; 16(5): 533-558.

54. Bierhaus A, Wolf J, Andrassy M, et al. A mechanism converting psychosocial stress into mononuclear cell activation. Proc Natl Acad Sci U S A. 2003; 100(4): 1920-5.

55. Canavan C, West J, Card T. The epidemiology of irritable bowe syndrome. Clin Epidemiol. 2014; 6: 71-80.

56. Mulak A, Bonaz B. Irritable bowel syndrome: a model of the brain-gut interactions. Med Sci Monit. 2004; 10(4): RA55-62.

57. Holtmann GJ, Ford AC, Talley NJ. Pathophysiology of irritable bowel syndrome. The Lancet Gastroenterology and Hepatology Elsevier Ltd. 2016; Vol. 1: 133-46. 
58. Wang F Bin, Powley TL. Vagal innervation of intestines: Afferent pathways mapped with new en bloc horseradish peroxidase adaptation. Cell Tissue Res. 2007; 329(2): 221-230.

59. Bonaz B, Sinniger V, Pellissier S. The Vagus Nerve in the NeuroImmune Axis: Implications in the Pathology of the Gastrointestinal Tract. Front Immunol. 2017; 8: 1452.

60. Zila I, Mokra D, Kopincova J, et al. Vagal-immune interactions involved in cholinergic anti-inflammatory pathway. Physiol Res. 2017; 66(Supplementum 2): S139-45.

61. Pellissier S, Bonaz B. The Place of Stress and Emotions in the Irritable Bowel Syndrome. Vitam Horm. 2017; 103: 327-54.

62. Houser MC, Tansey MG. The gut-brain axis: is intestinal inflammation a silent driver of Parkinson's disease pathogenesis? NPJ Park Dis. 2017; 3(1).

63. Boisse L, Chisholm SP, Lukewich MK, et al. Clinical and experimental evidence of sympathetic neural dysfunction during inflammatory bowel disease. Clin Exp Pharmacol Physiol. 2009; 36(10): 1026-33.

64. The FO, Boeckxstaens GE, Snoek SA, et al. Activation of the Cholinergic Anti-Inflammatory Pathway Ameliorates Postoperative Ileus in Mice. Gastroenterology. 2007; 133(4): 1219-28.

65. de Jonge WJ, van der Zanden EP, The FO, et al. Stimulation of the vagus nerve attenuates macrophage activation by activating the Jak2-STAT3 signaling pathway. Nat Immunol. 2005; 6(8): 844-51.

66. Ghia JE, Blennerhassett $\mathrm{P}$, Kumar-Ondiveeran $\mathrm{H}$, et al. The Vagus Nerve: A Tonic Inhibitory Influence Associated With Inflammatory Bowel Disease in a Murine Model. Gastroenterology. 2006; 131(4): 1122-1130

67. Bonaz B, Sinniger V, Hoffmann D, et al. Chronic vagus nerve stimulation in Crohn's disease: a 6-month follow-up pilot study. Neurogastroenterol Motil. 2016; 28(6): 948-53.

68. Matteoli G, Gomez-Pinilla PJ, Nemethova A, et al. A distinct vaga anti-inflammatory pathway modulates intestinal muscularis resident macrophages independent of the spleen. Gut. 2014; 63(6): 938-48.

69. Bonaz B, Bazin T, Pellissier S. The Vagus Nerve at the Interface of the Microbiota-Gut-Brain Axis. Front Neurosci. 2018; 12: 49.

70. Lacy BE, Weiser K, Lee R De. The treatment of irritable bowe syndrome. Therapeutic Advances in Gastroenterology. 2009; 2(4): 221-238.

71. Redgrave J, Day D, Leung $\mathrm{H}$, et al. Safety and tolerability of Transcutaneous Vagus Nerve stimulation in humans; a systematic review. Brain Stimul. 2018; 11(6): 1225-38.

72. Reid MC, Eccleston C, Pillemer K. Management of chronic pain in older adults. BMJ (Online) BMJ Publishing Group. 2015; Vol. 350.

73. Asmundson GJG, Katz J. Understanding the co-occurrence of anxiety disorders and chronic pain: State-of-the-art Depression and Anxiety. 2009; Vol. 26: 888-901.

74. Gureje 0, Von Korff M, Simon GE, et al. Persistent Pain and Well-being. JAMA. 1998; 280(2): 147.

75. Verhaak PFM, Kerssens JJ, Dekker J, et al. Prevalence of chronic benign pain disorder among adults: A review of the literature. Pain. 1998; 77(3): 231-239.

76. Wiech K. Deconstructing the sensation of pain: The influence of cognitive processes on pain perception. Science (80- ). 2016; 354(6312): 584-7.

77. Kirchner A, Birklein F, Stefan H, et al. [Vagus nerve stimulation - a new option for the treatment of chronic pain syndromes?]. Schmerz. 2001; 15(4): 272-7.

78. Borovikova LV, Ivanova S, Zhang $M$, et al. Vagus nerve stimulation attenuates the systemic inflammatory response to endotoxin. Nature. 2000; 405(6785): 458-62.
79. Junger $\mathrm{H}$, Sorkin LS. Nociceptive and inflammatory effects of subcutaneous TNFalpha. Pain. 2000; 85(1-2): 145-51.

80. Yunus MB. Central Sensitivity Syndromes: A New Paradigm and Group Nosology for Fibromyalgia and Overlapping Conditions, and the Related Issue of Disease versus Illness. Semin Arthritis Rheum. 2008; 37(6): 339-352.

81. Walker K, Dray A, Perkins M. Hyperalgesia in rats following intracerebroventricular administration of endotoxin: effect of bradykinin B1 and B2 receptor antagonist treatment. Pain. 1996; 65(2-3): 211-9.

82. Reeve AJ, Patel S, Fox A, et al. Intrathecally administered endotoxin or cytokines produce allodynia, hyperalgesia and changes in spinal cord neuronal responses to nociceptive stimuli in the rat. Eur J Pain. 2000; 4(3): 247-57.

83. Khasar SG, Miao FJ, Gear RW, et al. Vagal modulation of bradykinininduced mechanical hyperalgesia in the female rat. J Pain. 2003; 4(5): 278-83.

84. Saper CB. The central autonomic nervous system: conscious visceral perception and autonomic pattern generation. Annu Rev Neurosci. 2002; 25 : 433-69.

85. Janig W. The Integrative Action of the Autonomic Nervous System: Neurobiology of Homeostasis. Cambridge: Cambridge Unviersity Press. 2006.

86. Calvino B, Grilo RM. Central pain control. Joint Bone Spine Rev du Rhum. 2006; 73(1): 10-6.

87. Usichenko T, Hacker H, Lotze M. Transcutaneous auricular vagal nerve stimulation (taVNS) might be a mechanism behind the analgesic effects of auricular acupuncture. Brain Stimul. 2017; 10(6): 1042-4.

88. Multon S, Schoenen J. Pain control by vagus nerve stimulation: from animal to man...and back. Acta Neurol Belg. 2005; 105(2): 62-7.

89. Nishikawa Y, Koyama N, Yoshida Y, et al. Activation of ascending antinociceptive system by vagal afferent input as revealed in the nucleus ventralis posteromedialis. Brain Res. 1999; 833(1): 108-11.

90. Ren K, Randich A, Gebhart GF. Electrical stimulation of cervical vagal afferents. I. Central relays for modulation of spinal nociceptive transmission. J Neurophysiol. 1990; 64(4): 1098-114.

91. Chakravarthy K, Chaudhry H, Williams K, et al. Review of the Uses of Vagal Nerve Stimulation in Chronic Pain Management. Curr Pain Headache Rep. 2015; 19(12): 54

92. Chey WD, Kurlander J, Eswaran S. Irritable bowel syndrome: A clinical review. JAMA - Journal of the American Medical Association. American Medical Association. 2015; Vol. 313; 949-58.

93. Yunus MB. Central sensitivity syndromes: a new paradigm and group nosology for fibromyalgia and overlapping conditions, and the related issue of disease versus illness. Semin Arthritis Rheum. 2008; 37(6): 339-52.

94. Nicholson B, Verma S. Comorbidities in chronic neuropathic pain. Pain Med. 2004; 5 Suppl 1: S9-27.

95. Atkinson JH, Ancoli-Israel S, Slater MA, et al. Subjective sleep disturbance in chronic back pain. Clin J Pain. 1988; 4(4): 225-32.

96. Camilleri M. Management of the irritable bowel syndrome. Gastroenterology. 2001; 120(3): 652-68.

97. Fleming KC, Volcheck MM. Central Sensitization Syndrome and the Initial Evaluatin of a Patient with Fibromyalgia: A Review. Rambam Maimonides Med J. 2015; 6(2): e0020.

98. Bandelow B, Michaelis S, Wedekind D. Treatment of anxiety disorders Dialogues Clin Neurosci. 2017; 19(2): 93-106.

99. American Psychiatric Association. Diagnostic and Statistical Manual of Mental Disorders (5th Edition). American Journal of Psychiatry. 2013. 
100. Palsson OS, Whitehead WE, van Tilburg MA, et al. Rome IV Diagnostic Questionnaires and Tables for Investigators and Clinicians. Gastroenterology. 2016; 150(6): 1481-1491.

101. Bonaz B, Picq C, Sinniger V, et al. Vagus nerve stimulation: from epilepsy to the cholinergic anti-inflammatory pathway. Neurogastroenterol Motil. 2013; 25(3): 208-21.

102. Kraus T, Hosl K, Kiess O, et al. BOLD fMRI deactivation of limbic and temporal brain structures and mood enhancing effect by transcutaneous vagus nerve stimulation. J Neural Transm. 2007; 114(11): 1485-93.

103. Kraus T, Kiess O, Hosl K, et al. CNS BOLD fMRI effects of shamcontrolled transcutaneous electrical nerve stimulation in the left outer auditory canal - a pilot study. Brain Stimul. 2013; 6(5): 798804.

104. Mu Q, Bohning DE, Nahas Z, et al. Acute vagus nerve stimulation using different pulse widths produces varying brain effects. Biol Psychiatry. 2004; (8): 816-825.

105. Frøkjær JB, Bergmann S, Brock C, et al. Modulation of vagal tone enhances gastroduodenal motility and reduces somatic pain sensitivity. Neurogastroenterol Motil. 2016; 28(4): 592-598.

106. Panebianco M, Rigby A, Weston J, et al. Vagus nerve stimulation for partial seizures. Cochrane Database Syst Rev. 2015; (4): CD002896.

107. Groves DA, Brown VJ. Vagal nerve stimulation: a review of its applications and potential mechanisms that mediate its clinical effects. Neurosci Biobehav Rev. 2005; 29(3): 493-500.

108. Agnew WF, McCreery DB. Considerations for safety with chronically implanted nerve electrodes. Epilepsia. 1990; 31 Suppl 2: S27-32.

109. Yuan H, Silberstein SD. Vagus Nerve and Vagus Nerve Stimulation, a Comprehensive Review: Part III. Headache. 2016; 56(3): 479-90.

110. Howland RH. Vagus Nerve Stimulation. Curr Behav Neurosci Rep. 2014; 1(2): 64-73.

111. Spitzer RL, Kroenke K, Williams JB, et al. A brief measure for assessing generalized anxiety disorder: the GAD-7. Arch Intern Med 2006; 166(10): 1092-7.

112. Cleeland CS, Ryan KM. Pain assessment: global use of the Brief Pain Inventory. Ann Acad Med Singapore.1994; 23(2): 129-38.

113. Francis CY, Morris J, Whorwell PJ. The irritable bowel severity scoring system: a simple method of monitoring irritable bowel syndrome and its progress. Aliment Pharmacol Ther. 1997; 11(2): 395-402.

114. Cohen J. Statistical power analysis for the behavioral sciences (2nd ed.). Hillsdale, NJ: Lawrence Earlbaum Associates. Lawrence Earlbaum Associates. 1988

115. Denski KM, Labiner DM. Should I offer vagus nerve stimulation as part of my neurology practice? Neurol Clin Pr. 2014; 4(4): 313-8.

116. Mwamburi M, Liebler EJ, Tenaglia AT. Review of non-invasive vagus nerve stimulation (gammaCore): efficacy, safety, potential impact on comorbidities, and economic burden for episodic and chronic cluster headache. Am J Manag Care. 2017; 23(17 Suppl): S317-25.

117. Mwamburi M, Liebler EJ, Tenaglia AT. Cost-effectiveness of gammaCore (non-invasive vagus nerve stimulation) for acute treatment of episodic cluster headache. Am J Manag Care. 2017; 23(16 Suppl): S300-6.

118. Stefan H, Kreiselmeyer G, Kerling F, et al. Transcutaneous vagus nerve stimulation ( $t-V N S$ ) in pharmacoresistant epilepsies: a proof of concept trial. Epilepsia. 2012; 53(7): e115-8.

119. Morris 3rd GL, Mueller WM. Long-term treatment with vagus nerve stimulation in patients with refractory epilepsy. The Vagus Nerve Stimulation Study Group E01-E05. Neurology. 1999; 53(8): 1731-5.

120. Hord ED, Evans MS, Mueed S, et al. The effect of vagus nerve stimulation on migraines. J Pain. 2003; 4(9): 530-4.
121. Means-Christensen AJ, Roy-Byrne PP, Sherbourne CD, et al Relationships among pain, anxiety, and depression in primary care. Depress Anxiety. 2008; 25(7): 593-600.

122. Bair MJ, Robinson RL, Katon W, et al. Depression and pain comorbidity: a literature review. Arch Intern Med. 2003; 163(20): 2433-45.

123. Ligthart L, Visscher CM, van Houtem CM, et al. Comorbidity among multiple pain symptoms and anxious depression in a Dutch population sample. J Pain. 2014; 15(9): 945-55.

124. Neblett R, Cohen H, Choi Y, et al. The central sensitization inventory (CSI): Establishing clinically significant values for identifying central sensitivity syndromes in an outpatient chronic pain sample. J Pain. 2013 May; 14(5): 438-45.

125. Kirchner A, Birklein F, Stefan H, et al. Left vagus nerve stimulation suppresses experimentally induced pain. Neurology. 2000; (8)55 1167-1171.

126. Middlebrook N, Rushton AB, Heneghan NR, et al. Measures of central sensitisation and their measurement properties in the adult musculoskeletal trauma population: a protocol for a systematic review and data synthesis. BMJ Open. 2019; 9(3): e023204.

127. Fillingim RB, Loeser JD, Baron R, et al. Assessment of Chronic Pain: Domains, Methods, and Mechanisms. J Pain. 2016; 17 (9 Suppl): T1020.

128. Meregnani J, Clarencon D, Vivier M, et al. Anti-inflammatory effect of vagus nerve stimulation in a rat model of inflammatory bowel disease. Auton Neurosci. 2011; 160(1-2): 82-9.

129. Bernik TR, Friedman SG, Ochani M, et al. Cholinergic antiinflammatory pathway inhibition of tumor necrosis factor during ischemia reperfusion. J Vasc Surg. 2002; 36(6): 1231-6.

130. Lomarev M, Denslow S, Nahas Z, et al. Vagus nerve stimulation (VNS) synchronized BOLD fMRI suggests that VNS in depressed adults has frequency/dose dependent effects. J Psychiatr Res. 2002; 36(4): 219-27.

131. Vonck K, Boon P, D’Have M, et al. Long-term results of vagus nerve stimulation in refractory epilepsy. Seizure. 1999; 8(6): 328-34.

132. Henry TR, Bakay RA, Pennell PB, et al. Brain blood-flow alterations induced by therapeutic vagus nerve stimulation in partial epilepsy: II. prolonged effects at high and low levels of stimulation. Epilepsia. 2004; 45(9): 1064-70.

133. Straube A, Ellrich J, Eren 0, et al. Treatment of chronic migraine with transcutaneous stimulation of the auricular branch of the vagal nerve (auricular t-VNS): a randomized, monocentric clinical trial. J Headache Pain. 2015; 16(1).

134. Koopman FA, van Maanen MA, Vervoordeldonk MJ, et al. Balancing the autonomic nervous system to reduce inflammation in rheumatoid arthritis. J Intern Med. 2017; 282(1): 64-75.

135. Panebianco M, Zavanone $C$, Dupont $S$, et al. Vagus nerve stimulation therapy in partial epilepsy: a review. Acta Neurol Belg. 2016; 116(3): 241-8.

136. Tracey KJ. Physiology and immunology of the cholinergic antiinflammatory pathway. J Clin Invest. 2007; 117(2): 289-96.

137. Bonaz B, Sinniger V, Pellissier S. Anti-inflammatory properties of the vagus nerve: potential therapeutic implications of vagus nerve stimulation. J Physiol. 2016; 594(20): 5781-90.

138. Malik M, Camm AJ, Bigger JT, et al. Heart rate variability. Standards of measurement, physiological interpretation, and clinical use. European Heart Journal. 1996; 17(3): 354-381.

139. Hayano J. Introduction to heart rate variability. In: Clinical Assessment of the Autonomic Nervous System. 2016; 109-127. 
140. Ben-Tal A, Shamailov SS, Paton JFR. Evaluating the physiologica significance of respiratory sinus arrhythmia: Looking beyond ventilation-perfusion efficiency. J Physiol. 2012; 590(8): 1989-2008.

141. Yasuma F, Hayano JI. Respiratory Sinus Arrhythmia: Why Does the Heartbeat Synchronize with Respiratory Rhythm? Chest. 2004; 125(2): 638-690.

142. Kuo TBJ, Lai CJ, Huang YT, et al. Regression analysis between heart rate variability and baroreflex-related vagus nerve activity in rats. Cardiovasc Electrophysiol. 2005; 16(8): 864-869.

143. Shaffer F, Ginsberg JP. An Overview of Heart Rate Variability Metrics and Norms. Front Public Heal. 2017; 5(September): 1-17.

144. Kang JH, Kim JK, Hong SH, et al. Heart Rate Variability for Quantification of Autonomic Dysfunction in Fibromyalgia. Ann Rehabil Med. 2016; 40(2): 301-9.

145. Zamuner AR, Forti M, Andrade CP, et al. Respiratory Sinus Arrhythmia and its Association with Pain in Women with Fibromyalgia Syndrome. Pain Pr. 2016; 16(6): 704-11.

146. Staud R. Heart rate variability as a biomarker of fibromyalgia syndrome. Fut Rheumatol. 2008; 3(5): 475-83.
147. Pellissier S, Dantzer C, Canini F, et al. Psychological adjustment and autonomic disturbances in inflammatory bowel diseases and irritable bowel syndrome. Psychoneuroendocrinology. 2010; 35(5): 653-62.

148. Bandelow B, Baldwin D, Abelli M, et al. Biological markers for anxiety disorders, OCD and PTSD: A consensus statement. Part II: Neurochemistry, neurophysiology and neurocognition. World J Biol Psychiatry. 2017; 18(3): 162-214

149. Ha JH, Park S, Yoon D, et al. Short-term heart rate variability in older patients with newly diagnosed depression. Psychiatry Res. 2015; 226(2-3): 484-8.

150. Clancy JA, Mary DA, Witte KK, et al. Non-invasive vagus nerve stimulation in healthy humans reduces sympathetic nerve activity. Brain Stimul. 2014; 7(6): 871-7.

151. La Marca R, Nedeljkovic M, Yuan L, et al. Effects of auricular electrical stimulation on vagal activity in healthy men: evidence from a threearmed randomized trial. Clin Sci. 2010; 118(8): 537-546.

152. Laborde S, Mosley E, Thayer JF. Heart rate variability and cardiac vagal tone in psychophysiological research - Recommendations for experiment planning, data analysis, and data reporting. Front Psychol. 2017; 8(FEB): 1-18. 